\title{
Anomalous exciton Hall effect
}

\author{
V. K. Kozin, ${ }^{1,2}$ V. A. Shabashov, ${ }^{1,3}$ A. V. Kavokin, $,{ }^{4,5}, 6$ and I. A. Shelykh ${ }^{1,2}$ \\ ${ }^{1}$ ITMO University, Kronverkskiy prospekt 49, Saint Petersburg 197101, Russia \\ ${ }^{2}$ Science Institute, University of Iceland, Dunhagi-3, IS-107 Reykjavik, Iceland \\ ${ }^{3}$ St. Petersburg Academic University of the Russian Academy of Sciences, 194021 St. Petersburg, Russia \\ ${ }^{4}$ Westlake University, 18 Shilongshan Road, Hangzhou 310024, Zhejiang Province, China \\ ${ }^{5}$ Institute of Natural Sciences, Westlake Institute for Advanced Study, \\ 18 Shilongshan Road, Hangzhou 310024, Zhejiang Province, China \\ ${ }^{6}$ School of Physics and Astronomy, University of Southampton, \\ Highfield, Southampton SO17 1BJ, United Kingdom
}

\begin{abstract}
It is well known that electrically neutral excitons can still be affected by crossed electric and magnetic fields that make them move in a direction perpendicular to both fields. This effect is at the origin of the magnetic Stark effect for excitons and is described in terms of a synthetic gauge field. We show that a similar effect appears in the absence of external electric fields, in the case of scattering of an exciton flow by charge impurities in the presence of the external magnetic field. As a result, the exciton flow changes the direction of its propagation that may be described in terms of the Hall conductivity for excitons. We develop a theory of this effect, which we refer to as the anomalous exciton Hall effect, to distinguish it from the exciton Hall effect that arises due to the valley selective exciton transport in transition metal dichalcogenides. According to our estimations, the effect is relatively weak for optically active or bright excitons in conventional GaAs quantum wells, but it becomes significant for optically inactive or dark excitons, because of the difference of the lifetimes. This makes the proposed effect a convenient tool for spatial separation of dark and bright excitons.
\end{abstract}

Introduction. Thomas and Hopfield [1] were the first to point out that excitons propagating in the presence of an external magnetic field orthogonal to their velocity acquire stationary dipole polarisation perpendicular to both the magnetic field and their propagation direction. This is the manifestation of the magnetic Stark effect for excitons that was experimentally evidenced in a variety of semiconductor systems [2 4]. This effect is a manifestation of the Lorentz force that pulls an electron and a hole apart if an exciton as a whole particle moves in the presence of a magnetic field. Imamolu [5, 6] pointed out that once an exciton is placed in crossed electric and magnetic fields, it starts moving as a whole in the direction perpendicular to the directions of both fields, that leads to the renormalization of the excitonic dispersion semiconductor quantum wells or two-dimensional semiconductor crystals. Onga et al. [7] have recently reported the experimental observation of an exciton Hall effect in atomically thin layers of $\mathrm{MoS}_{2}$ that manifests itself in the appearance of an off-diagonal exciton conductivity in the presence of a magnetic field. The effect is caused by the strong spin-valley locking in monolayer transition metal dichalcogenides.

Here we propose one further step with respect to the three effects described above. We show that in conventional GaAs quantum wells containing charged impurities, an exciton flow may be reoriented in the real space due to the combined effect of the local electrostatic potential created by charged impurities and the magnetic field applied in normal to the plane direction. Conceptually, this effect is similar to the cross-field effect proposed by Imamolu [5, [6] and it manifests itself in a very simi- lar phenomenology to the exciton Hall effect studied by Onga et al. [7], however, it is different from both above mentionned effects as (i) an external electric field is not needed, (ii) spin-valley locking is not needed. To distinguish from the previous studies we refer to the effect we study as an anomalous exciton Hall effect.

We use the full microscopic description, based on the Lippmann-Schwinger equation in order to calculate the exciton scattering matrix on a single impurity, next we calculate the magnitude of the Hall angle for an exciton flow accounting for multiple scattering events and a finite exciton lifetime. We argue that the effect may have a significant magnitude in fluids of optically inactive, dark excitons due to their long lifetimes. On the other hand, the estimated Hall angle is only about 1 degree for short-living bright excitons in conventional GaAs-based quantum wells. This makes the anomalous Hall effect a powerful tool for spatial separation of dark and bright excitons.

The gauge field formalism. The concept of gauge fields is pivotal in high energy physics. In non-relativistic condensed matter physics gauge fields are also ubiquitous. The best known example is magnetic field $\mathbf{B}$. It is Abelian U(1) gauge field, which can be introduced into single particle Hamiltonian by substitution

$$
\hat{p}_{\mu} \rightarrow \hat{p}_{\mu}-q \hat{A}_{\mu}
$$

with $q$ being the electric charge of the particle, $A_{\mu}$ being components of the vector potential, $\mathbf{B}=\nabla \times \mathbf{A}(\mathbf{r})$. The presence of a magnetic field dramatically modifies the properties of the system, and leads to such fundamental phenomena as quantum Hall and Aharonov-Bohm ef- 
fects.

For neutral particles with $q=0$, such as photons, cold atoms, or excitons, magnetic field does not affect the orbital degree of freedom directly, and thus can not be considered as a real gauge field. However, if a particle possesses internal degrees of freedom, such as spin, polarization, or internal set of energy levels, creation of so called synthetic gauge fields becomes feasible. In general, they appear as consequences of a delicate interplay of the evolution of internal degrees of freedom with orbital dynamics in the adiabatic regime. In particular, for cold atoms both Abelian and non Abelian gauge potentials can be engineered by resonant drive of the system with spatially inhomogeneous laser beams [8]. For photons, the methods to create synthetic gauge fields include dynamic modulation [9], use of coupled optical resonators [10], engineering lattices with strain [11], or reciprocal metamaterials [12].

In condensed matter and semiconductor physics, the typical example of an electrically neutral particle is an exciton. Excitons can be created by optical excitation. They govern the optical response of semiconductor materials at low temperatures. In systems of the reduced dimensionality, such as monolayers of transition metal dichalcogenides (TMDs), excitonic features are highly pronounced and may be robust up to the room temperature [13]. The recent study of the electric field effect on the gauge fields for exciton strongly coupled to light (exciton-polaritons) [6] showed the high potentiality of the gauge field approach to the description of exciton and polariton dynamics in the presence of a magnetic field. In this Letter we demonstrate how the combination of the magnetic Stark effect [1] with excitonic scattering by an external potential, leads to the appearance of an effective $\mathrm{U}(1)$ gauge field acting on the motion of the exciton center of mass.

Phenomenological model. To reveal the nature of the proposed effects, we start with a simplified phenomenological model and assume that the motion of the center of mass of an exciton is decoupled from the internal dynamics of an electron and a hole forming it. We consider a $2 \mathrm{D}$ exciton confined in the $x y$-plane and subject to the external magnetic field directed along the $z$-axis. If the center of mass of the exciton is characterised by a non zero momentum $\mathbf{k} \neq 0$, the magnetic field acting on the electron and hole would dipole-polarize the exciton in the direction perpendicular to $\mathbf{k}$, so that the electric dipole moment of the exciton reads:

$$
\langle\hat{\mathbf{d}}\rangle=f(B)\left[\mathbf{e}_{z} \times \mathbf{k}\right]
$$

where $f(B)$ is a function of the magnetic field, which depends linearly on $B$ in the weak field limit, but becomes inversely proportional to $B$ at the large fields in the magneto-exciton regime $14-16$. At a small magnetic field one can find $f(B)$ by passing to the centerof-mass reference frame, where the magnetic field is ab- sent, but an electric field $\mathbf{E}^{\prime}=[\hbar \mathbf{k} \times \mathbf{B}] / M$ appears, here $M$ is the exciton mass. If this electric field is weak, then $\langle\hat{\mathbf{d}}\rangle=-e\langle\mathbf{r}\rangle=\alpha \mathbf{E}^{\prime}=\alpha[\hbar \mathbf{k} \times \mathbf{B}] / M$, where $\alpha=21 a_{\mathrm{B}}^{3} 4 \pi \epsilon_{0} \epsilon / 128$ is the $2 \mathrm{D}$ exciton polarizability [26], thus $f(B)=-\alpha \hbar B / M$. At strong magnetic fields, in the magneto-exciton regime, the dipole moment is given by [16] $\langle\hat{\mathbf{d}}\rangle=-e\langle\mathbf{r}\rangle=-e\left[\mathbf{e}_{z} \times \mathbf{k}\right] l_{B}^{2}$, where $l_{B}=\sqrt{\hbar /(e B)}$ is the magnetic length, thus $f(B)=-\hbar / B$.

The presence of impurities and fluctuations of the quantum well width leads to the appearance of the scattering potential $U_{\mathrm{sc}}(\mathbf{R})$ for excitons, here $\mathbf{R}$ denotes the position of the center-of-mass. Moreover, it generates some non-zero in-plane distribution of electric fields $\mathbf{E}(\mathbf{R})$, which can affect the exciton dipole moment given by Eq. (2) (see Fig. 11).

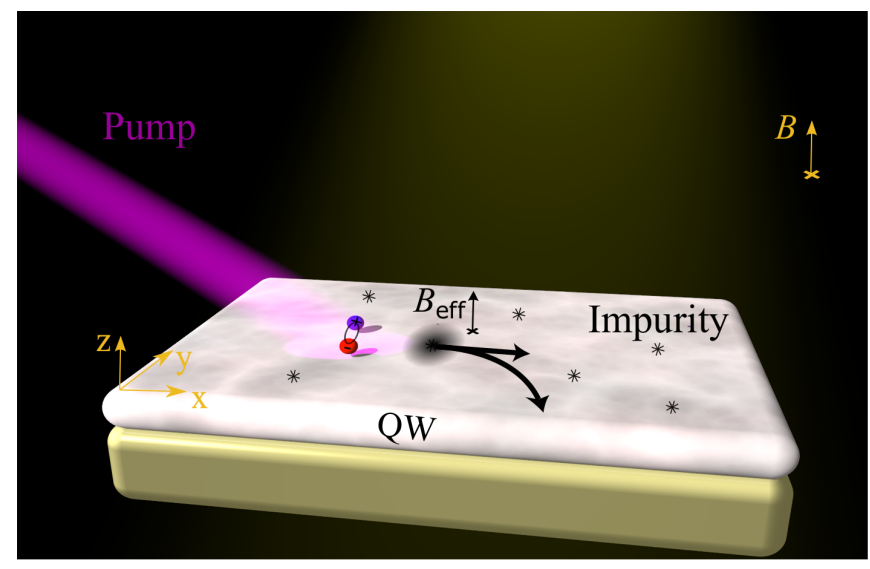

FIG. 1: The sketch of the system under consideration. Excitons created by the optical pump, travel in the plane of a disordered quantum well in the presence of a uniform magnetic field B, pointing perpendicularly to the quantum well. The magnetic field induces an in-plane dipole moment of the excitons which leads to the asymmetric scattering of the excitons by impurities. This problem can be mapped to the scattering of a charged particle by an impurity in the presence of the position-dependent synthetic magnetic field $\mathbf{B}_{\text {eff }}(\mathbf{R})$, depicted by the grey domain around the scatterer in the figure.

Using these assumptions we write down the model Hamiltonian of the system as:

$$
\hat{H}^{\mathrm{dip}}=\frac{\hat{\mathbf{p}}^{2}}{2 M}+\hat{V}^{\mathrm{dip}}=\frac{\hat{\mathbf{p}}^{2}}{2 M}+U_{s c}(\mathbf{R})-\frac{1}{2}(\hat{\mathbf{d}} \cdot \mathbf{E}+\mathbf{E} \cdot \hat{\mathbf{d}}),
$$

where $\hat{\mathbf{d}}=f(B)\left[\mathbf{e}_{z} \times \hat{\mathbf{k}}\right], M$ is the exciton mass, and $\hat{\mathbf{p}}=\hbar \hat{\mathbf{k}}$ is the exciton center-of-mass momentum operator. Note, that as exciton is a composite particle, there is no simple straightforward relation between the scattering potential and the electric field $\mathbf{E}=\mathbf{E}(\mathbf{R})$, producing by the scatterer. After some simple algebra this can be cast in form:

$$
\hat{H}^{\text {dip }}=\frac{1}{2 M}\left(\hat{\mathbf{p}}-e \mathbf{A}_{\mathrm{eff}}(\mathbf{R})\right)^{2}+U_{\mathrm{eff}}(\mathbf{R}),
$$


where $\mathbf{A}_{\text {eff }}(\mathbf{R})=M f(B)(\hbar e)^{-1}\left(\mathbf{e}_{x} E_{y}(\mathbf{R})-\mathbf{e}_{y} E_{x}(\mathbf{R})\right)$ is a synthetic vector potential, corresponding to the magnetic field $\mathbf{B}_{\text {eff }}(\mathbf{R})=-\mathbf{e}_{z} M f(B)(\hbar e)^{-1} \operatorname{div} \mathbf{E}(\mathbf{R})$ and $U_{\text {eff }}(\mathbf{R})=U_{\mathrm{sc}}(\mathbf{R})-M f^{2}(B)\left(2 \hbar^{2}\right)^{-1} \mathbf{E}^{2}(\mathbf{R})$ is an effective scalar potential. One can see that in order to have a non trivial synthetic gauge field, two conditions need to be fulfilled: (a) $B \neq 0$ and (b) the presence of an inhomogeneous electric field.

We consider first the scattering of an exciton by a single impurity that is characterized by the radial symmetry, i.e. we assume that $U_{s c}(\mathbf{R})=U_{s c}(R)$ and $\mathbf{E}(\mathbf{R})=$ $E(R) \mathbf{R} / R$. Then, assuming that the scattering is elastic, so that $|\mathbf{k}|=\left|\mathbf{k}^{\prime}\right|$, we obtain the following scattering matrix elements between the exciton states with the center-of-mass momenta $\mathbf{k}$ and $\mathbf{k}^{\prime}$

$$
\begin{aligned}
& V_{\mathbf{k}, \mathbf{k}^{\prime}}^{\operatorname{dip}}=U_{\mathrm{sc}}(\Delta \mathbf{k})+ \\
& \frac{i \hbar f(B)}{2 \pi} \frac{\left[\mathbf{k}^{\prime} \times \mathbf{k}\right]_{z}}{|\Delta \mathbf{k}|} \int_{0}^{\infty} R \mathrm{~d} R E(R) J_{1}(|\Delta \mathbf{k}| R) .
\end{aligned}
$$

Here we performed the integration over the polar angle, which yielded the Bessel functions, $\Delta \mathbf{k}=\mathbf{k}^{\prime}-\mathbf{k}$ is the transferred momentum. The first term $U_{\mathrm{sc}}(\Delta \mathbf{k}) \equiv$ $(2 \pi)^{-1} \int_{0}^{\infty} R \mathrm{~d} R U_{s c}(R) J_{0}(|\Delta \mathbf{k}| R)$ is real and it describes the normal symmetric scattering of an exciton by an impurity, while the second imaginary term accounts for the breaking of the time reversal symmetry by the magnetic field (as $V_{\mathbf{k}, \mathbf{k}^{\prime}}^{\text {dip }} \neq V_{-\mathbf{k}^{\prime},-\mathbf{k}}^{\text {dip }}$ ). This term gives rise to the asymmetric scattering (analogous to skew scattering) of excitons by the impurities and thus leads to the excitonic analogue of the anomalous Hall Effect.

Microscopic theory. In the presence of a magnetic field the center-of-mass motion and the internal motion are coupled, which has been neglected in the simplified qualitative model presented above. Now we proceed with the full microscopic model of exciton-impurity scattering in the presence of a magnetic field.

The Hamiltonian $\hat{H}_{\text {rel }}$ of relative motion of an $e-h$ pair characterized by the center-of-mass momentum $\hbar \mathbf{k}$ (where $\mathbf{k}$ is the wave vector) in the presence of a perpendicular magnetic field $B$ has the form [14, 18]

$$
\begin{aligned}
\hat{H}_{\mathrm{rel}}= & -\frac{\hbar^{2}}{2 \mu} \nabla_{\mathbf{r}}^{2}-\frac{i \hbar e B}{2}\left(\frac{1}{m_{e}}-\frac{1}{m_{h}}\right)\left[\mathbf{r} \times \nabla_{\mathbf{r}}\right]_{z}+ \\
& \frac{e^{2} B^{2}}{8 \mu} r^{2}+\frac{e \hbar}{M} \mathbf{B} \cdot[\mathbf{r} \times \mathbf{k}]-\frac{e^{2}}{4 \pi \varepsilon_{0} \varepsilon|\mathbf{r}|}+\frac{\hbar^{2} \mathbf{k}^{2}}{2 M}
\end{aligned}
$$

where $\mathbf{r}=\mathbf{r}_{e}-\mathbf{r}_{h}$ is the relative $e-h$ coordinate, and $\mu^{-1}=m_{e}^{-1}+m_{h}^{-1}$. Writing this expression we have taken advantage of the existence of an exact integral of motion, namely the magnetic center-of-mass momentum [18], defined by the operator $\hbar \hat{\mathbf{k}}=-i \hbar \nabla_{\mathbf{R}}-e \mathbf{A}(\mathbf{r})$, where $\mathbf{R}=\left(m_{e} \mathbf{r}_{e}+m_{h} \mathbf{r}_{h}\right) / M$ is the center-of-mass coordinate, $M=m_{e}+m_{h}$, and the vector potential is taken in the symmetrical gauge $\mathbf{A}(\mathbf{r})=\mathbf{B} \times \mathbf{r} / 2$. The exciton wave function has the form $\Psi_{\mathbf{k}}(\mathbf{R}, \mathbf{r})=$ $\exp \left\{i \frac{\mathbf{R}}{\hbar}[\hbar \mathbf{k}+e \mathbf{A}(\mathbf{r})]\right\} \Phi_{\mathbf{k}}(\mathbf{r})$, where $\Phi_{\mathbf{k}}(\mathbf{r})$ is the corresponding eingestate of the Hamiltonian above. An important point is that the wave function $\Phi_{\mathbf{k}}$ of the relative motion of an $e-h$ pair depends on the center-ofmass momentum $\hbar \mathbf{k}$ 18 i.e., the relative motion and the center-of-mass motion are coupled. The scattering matrix elements $V_{\mathbf{k}, \mathbf{k}^{\prime}}=\left\langle\Psi_{\mathbf{k}}|\hat{V}| \Psi_{\mathbf{k}^{\prime}}\right\rangle$ between the exciton states with the center-of-mass momenta $\mathbf{k}$ and $\mathbf{k}^{\prime}$ in the external potential $\hat{V}=V_{e}\left(\mathbf{r}_{e}\right)+V_{h}\left(\mathbf{r}_{h}\right)$ are given below.

Weak magnetic fields. In the weak-field limit, $l_{B} \gg$ $a_{\mathrm{B}}$, the problem can be treated analytically, here $l_{B}=$ $\sqrt{\hbar /(e B)}$ is the magnetic length and $a_{\mathrm{B}}=4 \pi \varepsilon_{0} \varepsilon \hbar^{2} /\left(\mu e^{2}\right)$ is the Bohr radius (the $1 s$ exciton radius is $a_{\mathrm{B}} / 2$ ). In this section we shall neglect excitonic transitions to the excited states of internal $e-h$ motion, i.e. the centerof-mass momentum $|\mathbf{k}| \ll a_{\mathrm{B}}^{-1}$. The ground-state wave function $\Phi_{\mathbf{k}}(\mathbf{r})$ is calculated in a magnetic field using the perturbation theory. The corresponding scattering matrix elements $V_{\mathbf{k}, \mathbf{k}^{\prime}}$ are obtained in Ref. [17] and read as follows

$$
\begin{aligned}
& V_{\mathbf{k}, \mathbf{k}^{\prime}}=\tilde{V}_{e}(\Delta \mathbf{k}) \mathcal{F}_{e}(\Delta \mathbf{k})+\tilde{V}_{h}(\Delta \mathbf{k}) \mathcal{F}_{h}(\Delta \mathbf{k})+ \\
& i\left[\mathbf{k}^{\prime} \times \mathbf{k}\right]_{z} a_{\mathrm{B}}^{2}\left(\frac{a_{\mathrm{B}}}{l_{B}}\right)^{2}\left(\tilde{V}_{e}(\Delta \mathbf{k}) \alpha_{e}-\tilde{V}_{h}(\Delta \mathbf{k}) \alpha_{h}\right) .
\end{aligned}
$$

Here $\tilde{V}_{j}(\mathbf{k})$ is the two-dimensional Fourier transform of the potentials $V_{j}(\mathbf{r}) \quad(j=e, h), \mathcal{F}_{e(h)}(\Delta \mathbf{k})=$ $\left[1-3 a_{\mathrm{B}}^{2} m_{h(e)}^{2}(\Delta \mathbf{k})^{2} /\left(32 M^{2}\right)+\beta_{e(h)}(\Delta \mathbf{k})^{2} a_{\mathrm{B}}^{2}\left(a_{\mathrm{B}} / \ell_{B}\right)^{4}\right]$, and $\Delta \mathbf{k}=\mathbf{k}^{\prime}-\mathbf{k}$ is the transferred momentum. In the derivation above only the essential terms of up to the second order in $B$ and the lowest orders in $|\mathbf{k}| a_{\mathrm{B}}$ are taken into account. The expressions for the dimensionless constants $\alpha_{e(h)}$ and $\beta_{e(h)}$ for a 2D Wannier-Mott exciton can be found in [17] (we corrected the typos in the original formulas): $\beta_{e(h)}=4^{-6} M^{-2}\left(105 m_{h(e)}^{2}-159 \mu^{2} / 2\right)$ and $\alpha_{e(h)}=-2 m_{h(e)} \kappa / M, \kappa=-21 \mu /\left(16^{2} M\right)$. Note that $\beta_{e}, \beta_{h}>0$ are positive, therefore, the exciton scattering cross-section increases with $B$ when $l_{B} \gg a_{\mathrm{B}}$. An important point is that for this scattering potential the time-reversal symmetry is broken $V_{\mathbf{k}, \mathbf{k}_{1}} \neq V_{-\mathbf{k}_{1},-\mathbf{k}}$, and the structure of $V_{\mathbf{k}, \mathbf{k}^{\prime}}$ resembles its counterpart (5) from the simplified phenomenological model. The exciton energy spectrum is given by

$$
\epsilon(\mathbf{k})=-\epsilon_{0}\left[1-\left(\frac{l_{2}}{l_{B}}\right)^{4}\right]+\frac{\hbar^{2} \mathbf{k}^{2}}{2 M}\left[1-2|\kappa|\left(\frac{a_{\mathrm{B}}}{l_{B}}\right)^{4}\right],
$$

where the parameter $l_{2}=(3 / 128)^{1 / 4} a_{\mathrm{B}}$ determines the diamagnetic shift. The first term represents the binding energy, whereas the second stands for the center-ofmass kinetic energy. Magneto-exciton regime. In the opposite regime, where the magnetic field is strong (i.e. $a_{\mathrm{B}} \gg l_{B}$ ), one can neglect the Coulomb interaction term 

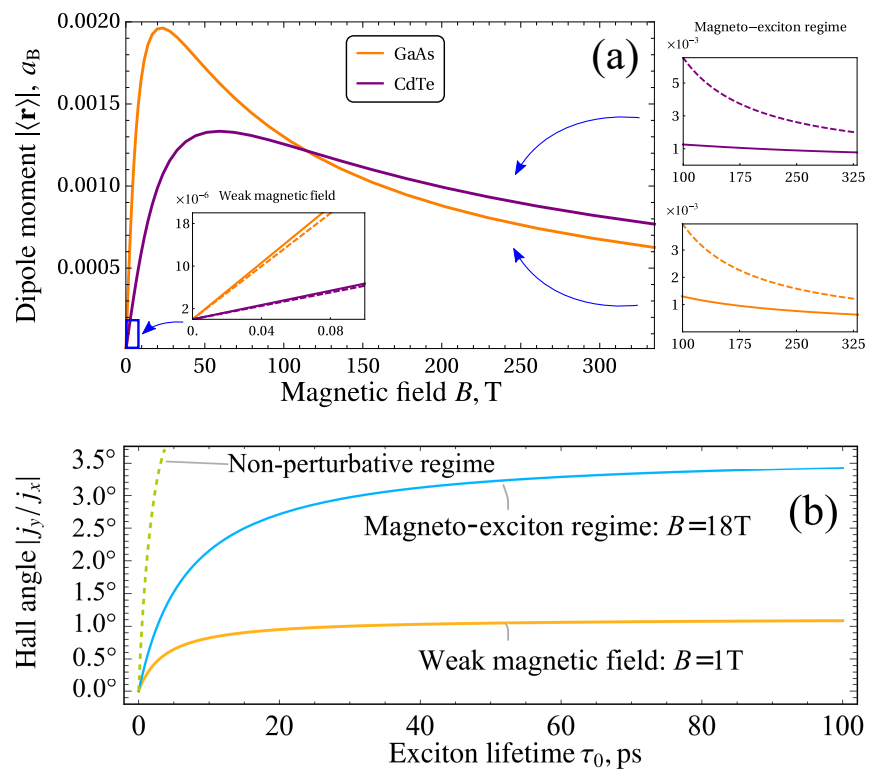

FIG. 2: (a) The dependence of the dipole moment (absolute value $|\langle\mathbf{r}\rangle|)$ on the magnetic field strength for GaAs and $\mathrm{CdTe}$ quantum wells. The solid lines are obtained from the numerically found eigenstates of Eq. (6), whereas the dashed lines are obtained, using the perturbation theory. The parameters of the plot are typical of GaAs quantum wells: the effective electron and hole masses $m_{e}=0.067 m_{e 0}, m_{h}=0.5 m_{e 0}$, the dielectric constant of the quantum well $\varepsilon=12.5$ (average dielectric constant of the surroundings). The exciton momentum corresponds to the wavelength of the absorbed photon $\lambda=860 \mathrm{~nm}$ as $|\mathbf{k}|=2 \pi \sin (\pi / 2-\pi / 8) / \lambda$, where the angle of incidence is set to $\pi / 2-\pi / 8$. The linear part of the curve describes the weak magnetic field limit, whereas the decaying tail corresponds to the magneto-exciton regime. (b) The Hall angle in a GaAs quantum well as a function of the exciton lifetime in two regimes: weak fields $a_{\mathrm{B}} / l_{B} \ll 1$ and the magneto-exciton regime $a_{\mathrm{B}} / l_{B} \gg 1$. The expected behavior in the non-perturbative regime $a_{\mathrm{B}} / l_{B} \sim 1$, where the Hall angle is expected to be the largest, is shown with the dashed curve.

in the Hamiltonian (6) and the exciton dynamics is governed by the magnetic field solely. In this regime, which is often referred to as the magneto-exciton regime, one can calculate the dispersion of the ground state (the electron and the hole occupy the lowest Landau level) of the system in the first order with respect to the $e-h$ Coulomb potential 25], which is now treated as a perturbation

$$
\epsilon(\mathbf{k})=\frac{1}{2} \hbar \omega_{\mathrm{C}}-\sqrt{\frac{\pi}{2}} \frac{e^{2}}{4 \pi \epsilon_{0} \epsilon l_{B}} e^{-\frac{k^{2} l_{B}^{2}}{4}} I_{0}\left(\frac{k^{2} l_{B}^{2}}{4}\right) .
$$

The corresponding impurity scattering matrix elements are given by

$$
\begin{array}{r}
V_{\mathbf{k}, \mathbf{k}^{\prime}}=\tilde{V}_{e}(\Delta \mathbf{k}) \exp \left(-\frac{i}{2}\left[\mathbf{k}^{\prime} \times \mathbf{k}\right]_{z} l_{B}^{2}-\frac{\Delta \mathbf{k}^{2} l_{B}^{2}}{4}\right)+ \\
\tilde{V}_{h}(\Delta \mathbf{k}) \exp \left(+\frac{i}{2}\left[\mathbf{k}^{\prime} \times \mathbf{k}\right]_{z} l_{B}^{2}-\frac{\Delta \mathbf{k}^{2} l_{B}^{2}}{4}\right),
\end{array}
$$

here $\omega_{\mathrm{C}}=e B / \mu$ is the cyclotron frequency.

Scattering rates. The impurity scattering matrix elements, discussing above for the two regimes, is the starting point for calculating the scattering $T$-matrix, which is defined as $T_{\mathbf{k}, \mathbf{k}^{\prime}}=\left\langle\Psi_{\mathbf{k}}|\hat{V}| \tilde{\Psi}_{\mathbf{k}^{\prime}}\right\rangle$, where $\hat{V}=$ $V_{e}\left(\mathbf{r}_{e}\right)+V_{h}\left(\mathbf{r}_{h}\right)$ is the impurity potential operator, $\left|\Psi_{\mathbf{k}}\right\rangle$ is the eigenstate of the free Hamiltonian $\hat{H}_{0}$, describing a $2 \mathrm{D}$ exciton in a magnetic field and $\left|\tilde{\Psi}_{\mathbf{k}^{\prime}}\right\rangle$ is the eigenstate of the full Hamiltonian $\hat{H}=\hat{H}_{0}+\hat{V}$. The $T$-matrix satisfies the Lippmann-Schwinger equation

$$
T_{\mathbf{k}, \mathbf{k}^{\prime}}=V_{\mathbf{k}, \mathbf{k}^{\prime}}+\int \frac{d^{2} \mathbf{g}}{(2 \pi)^{2}} \frac{V_{\mathbf{k}, \mathbf{g}} T_{\mathbf{g}, \mathbf{k}^{\prime}}}{E-\epsilon(\mathbf{g})+i 0},
$$

where $\epsilon(\mathbf{g})$ is the dispersion of the bare Hamiltonian $\hat{H}_{0}$ and it is given by Eq. (8) and Eq. (9) for the two regimes under consideration, respectively, and $E$ is the energy eigenvalue corresponding to $\left|\tilde{\Psi}_{\mathbf{k}^{\prime}}\right\rangle$. Two contributions can be distinguished in the square modulus of the $T$ matrix: $\nu_{0}^{2}\left|T_{\mathbf{k}, \mathbf{k}^{\prime}}\right|^{2}=\mathcal{G}_{\mathbf{k}, \mathbf{k}^{\prime}}+\mathcal{J}_{\mathbf{k}, \mathbf{k}^{\prime}}$, here $\mathcal{G}_{\mathbf{k}, \mathbf{k}^{\prime}} \equiv \mathcal{G}(\theta)=$ $\mathcal{G}(-\theta), \mathcal{J}_{\mathbf{k}, \mathbf{k}^{\prime}} \equiv \mathcal{J}(\theta)=-\mathcal{J}(-\theta)$ are dimensionless symmetric and asymmetric contributions to the scattering rate, respectively, $\theta=\varphi-\varphi^{\prime}$ is the scattering angle, $\varphi, \varphi^{\prime}$ are the polar angles of $\boldsymbol{k}, \boldsymbol{k}^{\prime}$, and $\nu_{0}=M /\left(2 \pi \hbar^{2}\right)$ is the $2 \mathrm{D}$ density of states of free particles with parabolic dispersion, which is introduced here to write the scattering matrices in dimensionless form. The density of states is defined as $\nu(\mathbf{k})=|\partial \epsilon(\mathbf{k}) / \partial k|^{-1} k /(2 \pi)$. Here we restrict ourselves to the case $|\mathbf{k}|=\left|\mathbf{k}^{\prime}\right|$, which corresponds to the elastic scattering. It is the asymmetric part $\mathcal{J}_{\mathbf{k}, \mathbf{k}^{\prime}}$ of exciton scattering by impurities that gives rise to the Hall current. The properties of $\mathcal{J}_{\mathbf{k}, \mathbf{k}^{\prime}}$ are discussed in details in Ref. [19, 20, 23].

The elastic scattering rate $W_{\mathbf{k}, \mathbf{k}^{\prime}}$ from $\mathbf{k}^{\prime}$ to $\mathbf{k}$ state is expressed with use of the Fermi golden rule $W_{\mathbf{k}, \mathbf{k}^{\prime}}=$ $2 \pi \hbar^{-1} n_{\text {imp }}\left|T_{\mathbf{k}, \mathbf{k}^{\prime}}\right|^{2} \delta\left(\epsilon_{\mathbf{k}}-\epsilon_{\mathbf{k}^{\prime}}\right)$, where $n_{\text {imp }}$ is the surface density of impurities. The presence of the magnetic field breaks the time inversion symmetry in the problem and leads to the fact that scattering rates $W_{\mathbf{k}, \mathbf{k}^{\prime}}$ and $W_{\mathbf{k}^{\prime}, \mathbf{k}}$ become non-equivalent and thus a non-zero Hall contribution $\mathcal{J}_{\mathbf{k}, \mathbf{k}^{\prime}}$ emerges.

Let us assume that the impurity potential is described by the Coulomb-like potential [24] $V_{e}(r)=-V_{h}(r)=$ $-e q_{\text {imp }} /\left(4 \pi \varepsilon_{0} \varepsilon r\right)$. The Lippmann-Schwinger equation can not be treated perturbatively in our system, which is why we solve this integral equation (11) numerically. Note that for $B=0$ it follows that $V_{\mathbf{k}, \mathbf{k}^{\prime}}$ is real (see Eq. (7)), therefore as expected, $\mathcal{J}_{\mathbf{k}, \mathbf{k}^{\prime}}=0$, i.e. scattering is purely symmetrical.

Having solved the Lippmann-Schwinger equation numerically in the two regimes under consideration, one may proceeds with calculating the Hall current appearing in the system. We study the classical transport regime $|\mathbf{k}| l \gg 1$, where $l$ is the exciton mean free path. We use the semiclassical Boltzmann equation for the occupation 
numbers of exciton states $n_{\mathbf{k}}$ having wave vectors $\mathbf{k}$

$$
\frac{d n_{\mathbf{k}}}{d t}=P_{\mathbf{k}}-\Gamma n_{\mathbf{k}}+\int \frac{d^{2} \mathbf{k}^{\prime}}{(2 \pi)^{2}}\left(W_{\mathbf{k}, \mathbf{k}^{\prime}} n_{\mathbf{k}^{\prime}}-W_{\mathbf{k}^{\prime}, \mathbf{k}} n_{\mathbf{k}}\right),
$$

here $P_{\mathbf{k}}$ is the coherent pump term, $\Gamma=1 / \tau_{0}$ is the particle decay rate, which is assumed to be independent of k. We consider the case where the equilibrium is reached and $n_{\mathbf{k}}$ doesn't depend on time, thus the time derivative term will be omitted. Then, performing the integration over the absolute value of $\mathbf{k}^{\prime}$, the Boltzmann kinetic equation can be rewritten as

$$
0=P_{\mathbf{k}}-\Gamma n_{\mathbf{k}}+\int d \varphi^{\prime}\left(w_{\mathbf{k}, \mathbf{k}^{\prime}} n_{\mathbf{k}^{\prime}}-w_{\mathbf{k}^{\prime}, \mathbf{k}} n_{\mathbf{k}}\right),
$$

where $w_{\mathbf{k}, \mathbf{k}^{\prime}}=n_{\text {imp }} \nu_{0} /\left(\hbar \nu(\mathbf{k})^{2}\right)\left(\mathcal{G}_{\mathbf{k}, \mathbf{k}^{\prime}}+\mathcal{J}_{\mathbf{k}, \mathbf{k}^{\prime}}\right)$ and the exciton density of states $\nu(\mathbf{k})=|\partial \epsilon(\mathbf{k}) / \partial k|^{-1} k /(2 \pi)$. Let us assume the in-plane wave vector $\mathbf{k}_{0}$ of the pump is pointing along the $x$-axis, i.e. $\mathbf{k}_{0}=\left(k_{0}, 0\right)^{T}$, which implies $P_{\mathbf{k}}=P_{0} \delta\left(\mathbf{k}-\mathbf{k}_{0}\right)=\left(P_{0} / k\right) \delta\left(k-k_{0}\right) \delta(\varphi)$ and we note that this function is even with respect to $\varphi$. Since $w_{\mathbf{k}, \mathbf{k}^{\prime}}$ depends on the difference between the momentum angles, to perform the integration over $\varphi^{\prime}$ in the collision integral, the particle density $n_{\mathbf{k}}$ and the scattering rate $w_{\mathbf{k}, \mathbf{k}^{\prime}}$ should be expanded in cylindrical harmonics $\cos m \varphi$, $\sin m \varphi$, where $m \geq 0$ is integer. Assuming that only the dipole type of anisotropy of the momentum-space distribution function is significant, we represent $n_{\mathbf{k}}=$ $n_{0}(k)+\delta n(\mathbf{k})$, where $\delta n(\mathbf{k})=n_{+}(k) \cos \varphi+n_{-}(k) \sin \varphi$, $n_{0}(k)$ is the isotropic part of the distribution function which depends only on energy. The coefficients $n_{ \pm}(k)$ are to be found. Substituting this decomposition into the kinetic equation (13) and integrating over $\varphi^{\prime}$ in the collision term, we obtain

$$
\begin{aligned}
& 0=\frac{P_{0}}{k} \delta\left(k-k_{0}\right) \delta(\varphi)+\cos \varphi\left(\Omega(k) n_{-}(k)-\frac{n_{+}(k)}{\tau(k)}\right) \\
& -\sin \varphi\left(\Omega(k) n_{+}(k)+\frac{n_{-}(k)}{\tau(k)}\right)-\frac{n_{\mathbf{k}}}{\tau_{0}}
\end{aligned}
$$

where $\tau(k)$ is given by the symmetric scattering term $\tau(k)^{-1}=n_{\text {imp }} \nu_{0} /\left(\hbar \nu(\mathbf{k})^{2}\right) \int_{0}^{2 \pi} \mathcal{G}_{\mathbf{k}, \mathbf{k}^{\prime}}(1-\cos \theta) d \theta$, here $\theta=\varphi-\varphi^{\prime}$ is the scattering angle. The factor $\Omega(k)$ is determined by the asymmetric scattering term $\Omega(k)=-n_{\text {imp }} \nu_{0} /\left(\hbar \nu(\mathbf{k})^{2}\right) \int_{0}^{2 \pi} \mathcal{J}_{\mathbf{k}, \mathbf{k}^{\prime}} \sin \theta d \theta$, and it mixes the even $n_{+}(k)$ and odd $n_{-}(k)$ contributions to the density distribution, yielding a Hall current in the transverse $y$-direction. Using the orthogonality of $\sin \varphi$ and $\cos \varphi$, the kinetic equation is readily solved, yielding $n_{-}(k)=-\Omega(k) \tau_{\text {tot }}(k) n_{+}(k)$. Here we introduced the total relaxation time $\tau_{\text {tot }}(k)=\left(\tau_{0}^{-1}+\tau(k)^{-1}\right)^{-1}$. The Hall angle, defined as the ratio of the Hall current $j_{y}$ and the longitudinal current $j_{x}$, is $j_{y} / j_{x}=-\Omega\left(k_{0}\right) \tau_{\text {tot }}\left(k_{0}\right)$, where $j_{x, y}=\int \hbar k_{x, y} \delta n(\mathbf{k}) d^{2} \mathbf{k} /(2 \pi)^{2}$. This quantity can be estimated for the typical parameters of doped GaAs quantum wells $n_{\text {imp }} \approx 10^{11} \mathrm{~cm}^{-2}, k_{0} \approx 7 \cdot 10^{6} \mathrm{~m}^{-1}$ (see the caption of Fig. 21), $\varepsilon=12.5$, the charge of the impurity $q_{\mathrm{imp}}=e$, and $\tau_{0} \approx 10 \mathrm{ps}$ (for bright excitons) in the two regimes. At a weak magnetic field $B=1 \mathrm{~T}$ the numerical solution of the Lippmann-Schwinger equation with the kernel (7), yields $\tau\left(k_{0}\right) \approx 3.8 \mathrm{ps}$ and $j_{y} / j_{x}=-\Omega\left(k_{0}\right) \tau_{\text {tot }}\left(k_{0}\right) \approx-0.8 \%$. At a strong magnetic field $B=18 \mathrm{~T}$ we solve the Lippmann-Schwinger integral equation numerically with the magneto-exciton kernel (10), which yields $j_{y} / j_{x}=-\Omega\left(k_{0}\right) \tau_{\text {tot }}\left(k_{0}\right) \approx-1.8 \%$. The negative sign of the Hall angle $\left(j_{y} / j_{x}\right)$ indicates that the Hall current is antiparallel to the $y$-direction. One may expect, by looking at Fig. 2(a), that at intermediate magnetic field strengths (about $B \approx 10 \mathrm{~T}$ ) the Hall angle would be significantly larger, as the exciton dipole moment reaches its largest value in this non-perturbative regime, while still not switching to the magneto-exciton regime. We also note, that the range of magnetic fields corresponding to the largest dipole moment is easily achievable experimentally, which makes the observation of the predicted phenomenon realistic. The dependence of the Hall angle on the exciton lifetime is shown in Fig. 2(b). Clearly, for dark excitons whose lifetime is significantly larger than the lifetime of bright excitons, the Hall angle is notably larger. This shows that the anomalous exciton Hall effect may be used as a tool for spatial separation of dark and bright excitons.

Conclusions. In conclusion, we demonstrated that the magnetic Stark effect for 2D excitons may lead to the emergence of an effective U(1) gauge field. This field can result in the excitonic analogue of the anomalous Hall effects. For the later we presented a detailed microscopic description of the scattering mechanism and analyzed the transport properties, showing that the effect can be observed experimentally in conventional GaAs quantum wells and that it is much stronger for dark than bright excitons.

The work of V.K.K. was supported by Russian Science Foundation, Grant No. 19-72- 20120. I.A.S. thanks Ministry of Education and Science of Russian Federation, Project 14.Y26.31.0015, and Icelandic Research Fund (Rannis), project "Hybrid Polaritonics". AK acknowledges Project No. 041020100118 and Program 2018R01002 supported by Leading Innovative and Entrepreneur Team Introduction Program of the Zhejiang Province.

[1] D. G. Thomas, J. J. Hopfield, Phys. Rev. 124, 657 (1961).

[2] M. Lafrentz, D. Brunne, B. Kaminski,V. V. Pavlov, A.V.Rodina, R. V. Pisarev, D. R. Yakovlev, A. Bakin, and M. Bayer, Phys. Rev. Lett. 110, 116402 (2013).

[3] Y. J. Chen, E. S. Koteles, B. S. Elman, and C. A. Armiento, Phys. Rev. B 36, 4562 (1987).

[4] P. Andreakou, A. V. Mikhailov, S. Cronenberger, D. 
Scalbert, A. Nalitov, A. V. Kavokin, M. Nawrocki, L. V. Butov, K. L. Campman, A. C. Gossard, and M. Vladimirova, Phys. Rev. B 93, 115410 (2016).

[5] A. Imamolu, Phys. Rev. B 85, 14285 (1996).

[6] H-T. Lim, E. Togan, M. Kroner, J. Miguel-Sanchez and A. Imamolu, Nature Communications 8, 14540 (2017).

[7] M. Onga, Y. Zhang, T. Ideue \& Y. Iwasa, Nature Materials 16, 1193 (2017).

[8] Jean Dalibard, Fabrice Gerbier, Gediminas Juzelinas, and Patrik hberg, Rev. Mod. Phys. 83, 1523 (2011).

[9] K. Fang, Z. Yu, S. Fan, Nat. Photon. 6, 782 (2012).

[10] M. Hafezi, E. A. Demler, M. D. Lukin, J. M. Taylor, Nat. Phys. 7, 907912 (2011).

[11] M. C. Rechtsman, et al., Nat. Photon. 7, 153 (2013).

[12] F. Liu, T. Xu, S. Wang, Z. H. Hang, J. Li, Adv. Opt. Mater. 7, 1801582 (2019).

[13] Gang Wang, Alexey Chernikov, Mikhail M. Glazov, Tony F. Heinz, Xavier Marie, Thierry Amand, and Bernhard Urbaszek, Rev. Mod. Phys. 90, 021001 (2018).

[14] I. V. Lerner, Y.E. Lozovik, Zh. Eksp. Theor. Phys. 78, 1167 [Sov. Phys. JETP 51, 588] (1978)

[15] L. V. Butov, C. W. Lai, D. S. Chemla, Yu. E. Lozovik, K. L. Campman, and A. C. Gossard Phys. Rev. Lett. 87, 216804 (2001)
[16] Yu. E. Lozovik, I. V. Ovchinnikov, S. Yu. Volkov, L. V. Butov, and D. S. Chemla Phys. Rev. B 65, 235304 (2002)

[17] Arseev, P.I., Dzyubenko, A.B. Exciton, J. Exp. Theor. Phys. 87, 200209 (1998)

[18] L. P. Gorkov and I. E. Dzyaloshinskii, Zh. Eksp. Teor. Fiz. 53, 717 (1967) [Sov. Phys. JETP 26, 449 (1968)].

[19] Denisov, K. S., Rozhansky, I. V., Averkiev, N. S. et al., Sci Rep 7, 17204 (2017).

[20] K. S. Denisov, I. V. Rozhansky, N. S. Averkiev, and E. Lhderanta, Phys. Rev. Lett. 117, 027202 (2016)

[21] F. E. Meijer, A. F. Morpurgo, and T. M. Klapwijk Phys. Rev. B 66, 033107 (2002)

[22] V. K. Kozin, I. A. Shelykh, A. V. Nalitov, and I. V. Iorsh Phys. Rev. B 98, 125115 (2018)

[23] K. S. Denisov, I. V. Rozhansky, N. S. Averkiev, and E. Lhderanta Phys. Rev. B 98, 195439 (2018)

[24] S. Fraizzoli, F. Bassani, and R. Buczko Phys. Rev. B 41, 5096 (1990)

[25] Alexander B. Dzyubenko and Gerrit E. W. Bauer Phys. Rev. B 51, 14524 (1995)

[26] Thomas G. Pedersen, Exact polarizability of lowdimensional excitons, Solid State Communications, Volume 141, Issue 10, 2007, 